\title{
Development of a Simple Efficient Controller to Improve Response of MR Fluid Brakes
}

\author{
Injun Hwang1), Sooho Woo ${ }^{1)}$, Soongeul Lee2), Ihnsik Weon³)
}

\begin{abstract}
In this paper, a simple efficient controller was developed to improve torque response characteristics of MR fluid brakes over time. Due to the nature of MR fluid brakes with different characteristics depending on the applied current, conventional voltage application methods are difficult to use when linear control of residual magnetic and brake torque is required. The proposed controller is intended to be used to propose a method for adjusting the signals of Enable_VH, MR_on, and Fast_off over time to increase the rate in the rise and fall of torque over generation torque control and time through voltage control applied to MR fluid brakes. Arduino Uno is used as the control board for the control and current sensors and torque sensors were used to check torque response characteristics. The control program used LabView to measure the current and torque applied to the MR fluid brakes. Experimental and comparative analyses were carried out using the controller proposed in this paper and the rated voltage was applied to determine the effect of the simple efficient controller on torque generation of MR fluid brake. Based on the results of the Rise Time and Fall Time of the MR fluid brakes, the efficiency of the proposed controller and comparative analysis of the response characteristics of the MR fluid brakes according to the input current could be verified.

Keywords: MR Fluid Brake, Response Characteristics, Simple Efficient Controller, LabView, Torque Control
\end{abstract}

\section{Introduction}

Recently, studies have been actively conducted on high-efficiency and high-torque actuators for applications to various fields and mechanical device actuators. MR fluid brakes are a type of actuator that can transmit power using electric signals and are easy to miniaturize due to its simple structural advantages, currently many studies are underway[1-3]. MR fluid brakes are devices capable of braking by means of the coupling force of MR fluid molecules according to

Received(October 14, 2019), Review Result(1st: November 01, 2019, 2nd: December 12, 2019), Accepted(February 20, 2020)

1) (Student) 17104 Dept. Mechanical Engineering, KyungHee Univ., 1732 Dukyoungdaero, Giheung-gu, Yongin, Korea email: hollis00@khu.ac.kr, woosh828@khu.ac.kr

2) (Professor, Corresponding Author) 17104 Dept. Mechanical Engineering, KyungHee Univ., 1732 Dukyoungdaero, Giheung-gu, Yongin, Korea

email: sglee@khu.ac.kr

3) (Ph. D) 3488 Unmanned/Robotic systems, LIG Nex1, 333., Pangyo-ro, Bundang-gu, Seongnam, Korea email: ihnsik.weon@lignex1.com 
the strength of the magnetic field formed. Unlike normal brakes, a simple structure without a wetted part is advantageous for miniaturization that offers high power ratio per unit volume/weight[4-6].

In most previous studies, constant voltage direct current voltage had been used so that the current applied to the solenoid coil of the MR fluid brake is simply on/off, so that the torque of the MR fluid brakes is between 0 and maximum values[7-9]. However, due to the nature of MR fluid brakes having different characteristics depending on the applied current, these fragmentary methods are difficult to use when a linear control of magnet-related residual magnetism and brake torque are required[9-12]. These fragmentary methods are also inefficient in terms of the response characteristics of torque generation overtime of the MR fluid brakes.

In this paper, we developed a controller that can increase the generated torque control and the torque rising and falling speed efficiency over time through using current control. The efficiency of the controller was verified by comparing and analyzing the response characteristics of the MR fluid brakes according to the input current.

\section{Simple Efficient Controller Block Diagram and Control Theory}

The block diagram of the simple efficient controller used in improving the response characteristics of MR fluid brakes is shown in [Fig. 2] below. This structure controls the voltage applied to the MR fluid brakes by adjusting the signals of Enable_VH, MR_on, and Fast_off.

The Enable_VH signal flows at VH (maximum allowable voltage), and the MR_on signal controls the current flow of the MR fluid brake. Additionally, the Fast_off signal acts as a quick removal of the residue current, which is inside the circuit by giving a reverse current. The voltage applied to the MR fluid brake over time is $\mathrm{VH}$ for $\triangle$ on, $\mathrm{VN}$ (rated voltage) for $t_{V N}$, and $\mathrm{VL}$ for $\Delta o f f$. With the high voltage on the $\mathrm{VH}$ for $\Delta$ on the inflation rate of torque therefore increases.

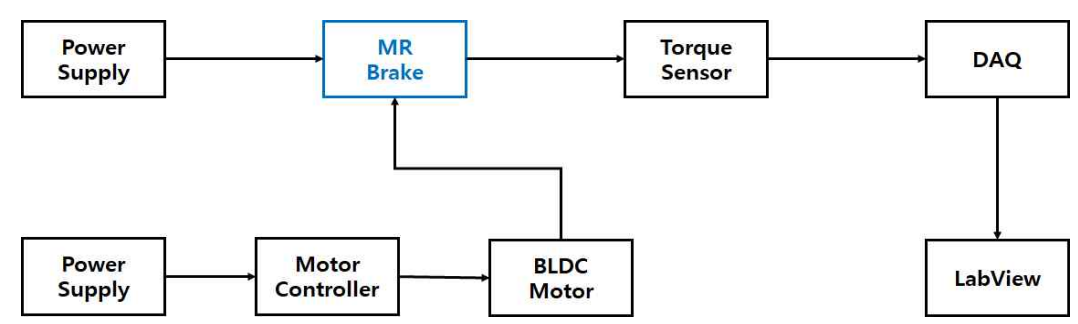

[Fig. 1] General Switching Controller Block Diagram 


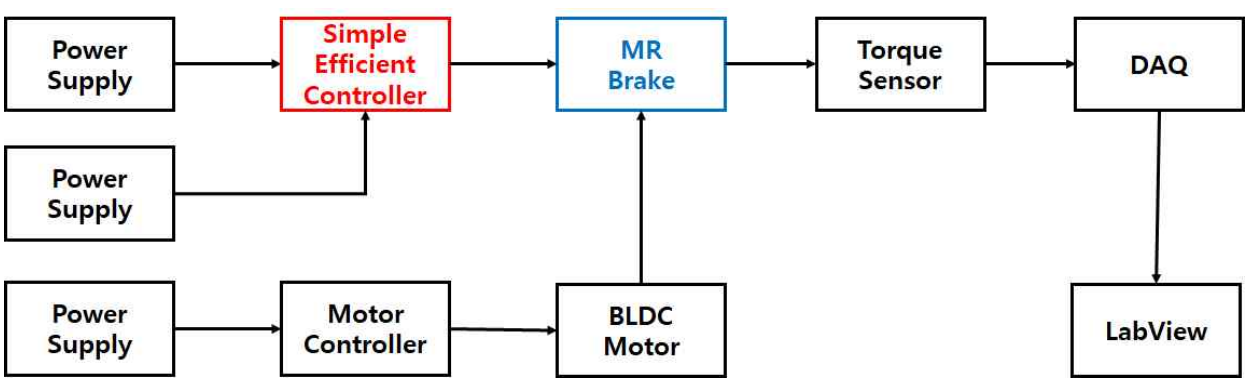

[Fig. 2] Simple Efficient Controller Block Diagram

Because Rise Time is affected by the time of the $\triangle$ on authorizing $\mathrm{VH}, \triangle$ on's decision is an important factor. $\triangle o n$ is determined by the following formula and can finally be expressed as Formula (6). In the formula below, $I_{\max }$ represents the maximum permitted current of the coil, $R$ represents the resistance of the coil, and $L$ represents the magnetic inductance.

$$
\begin{aligned}
& i=\frac{V H}{R}\left(1-e^{-\Delta o n / \tau}\right)=I_{\max } \\
& I_{\max } \times \frac{R}{V H}=1-e^{-\Delta o n / \tau} \\
& 1-I_{\max } \times \frac{R}{V H}=e^{-\Delta o n / \tau} \\
& \ln \left(1-I_{\max } \times \frac{R}{V H}\right)=-\frac{\Delta o n}{\tau} \\
& \triangle \text { on }=-\tau \times \ln \left(1-I_{\max } \times \frac{R}{V H}\right) \\
& \Delta \text { on }=-\frac{L}{R} \times \ln \left(1-I_{\max } \times \frac{R}{V H}\right)
\end{aligned}
$$

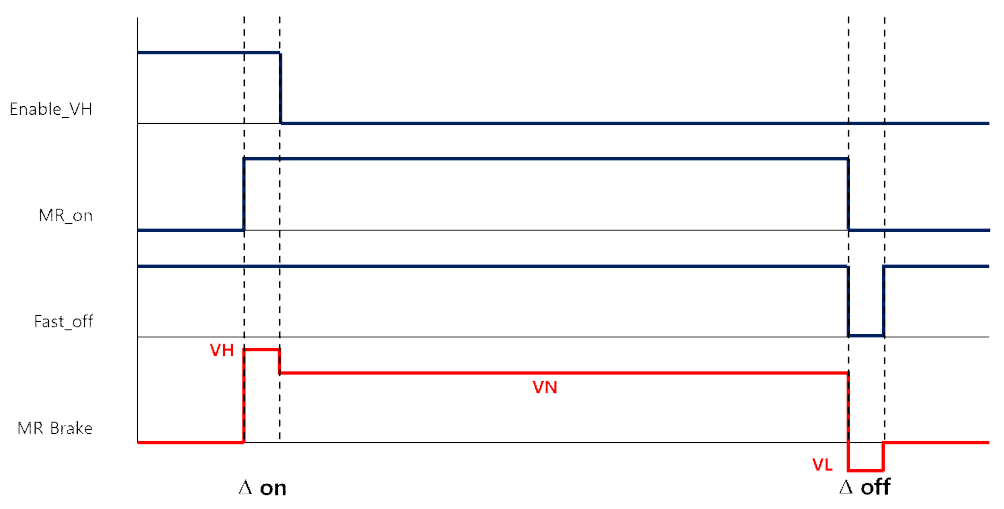

[Fig. 3] MR Fluid Brake Applied Voltage according to Signal 


\section{Experiments and Results}

\subsection{Experimental Configuration}

The control board used in this experiment was the Arduino Uno. Current sensors (WCS2702) and torque sensors (SI 580-20) were used to obtain current and torque data acting on MR fluid brakes. The LabView program was used to collect data. The analysis was conducted on the 10 - $90 \%$ section between the maximum and minimum torque values. The reason is to minimize the noise impact of the torque sensor. The rise time of the torque when the power supply is turned on is called RiseTime. And when the power supply is turned off, the drop time of the torque is called FallTime.

The experiment with General-Switching-Controller was conducted by simply on-off the rated voltage through direct connection between the power supply and the MR fluid brake. The General-Switching-Controller experiment proposed in this paper was conducted by entering different voltages of $\mathrm{VH}, \mathrm{VN}$ and $\mathrm{VL}$ over time as shown in [Fig. 3]. The Rise and Fall Time of the two experiments were compared to confirm the increase in efficiency with respect to torque response over time of MR fluid brakes by applying General-Switching-Controller.

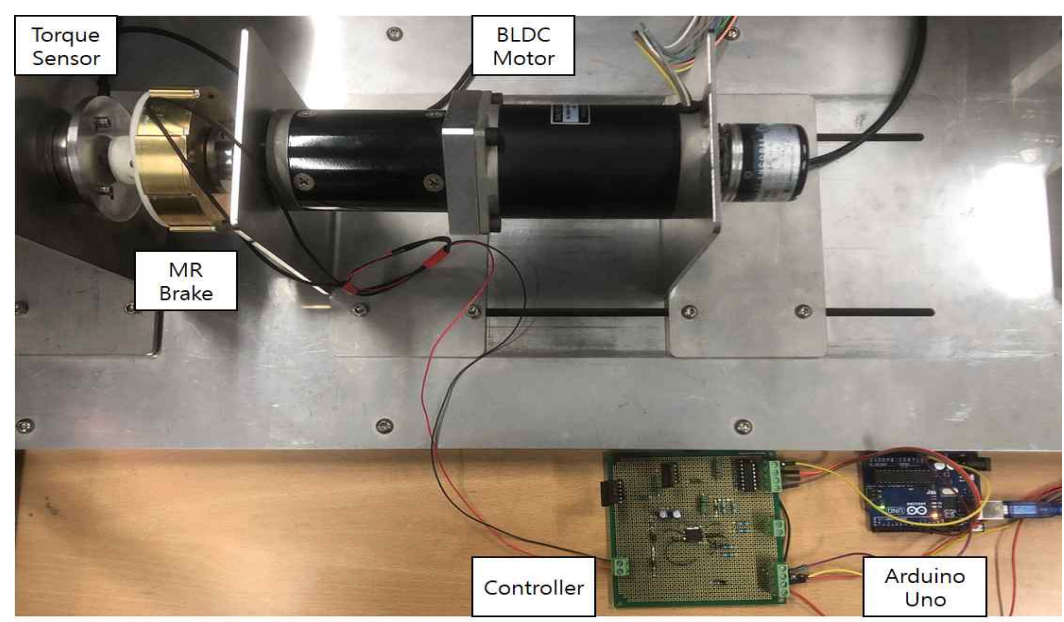

[Fig. 4] TestBed for Torque Response Test

\subsection{The Result of Experiment}

The results of the experiments conducted in this paper are shown in [Fig. 5]. It shows the 
rapid increase and stabilization of torque through the $\mathrm{VH}$ input for $\triangle$ on period of the simple efficient controller use.

For the input current of $0.2 \mathrm{~A}$, the Rise Time was measured $186.9 \mathrm{~ms}$ and the Fall Time was measured as $173.4 \mathrm{~ms}$ when the rated voltage was entered. The Rise Time for the use of simple efficient controllers was measured at $42.2 \mathrm{~ms}$ and the Fall Time at $175.2 \mathrm{~ms}$. These results show that the use of simple efficient controllers has accelerated the response characteristics of the Rise Time.

[Table 1] also shows the response characteristics according to the input current. Through these results, it can be observed that the larger the input current value, the faster the response becomes. This shows that the higher the current and voltage applied to the MR fluid brakes, the greater the rate of torque generation over time. It can also be verified that the use of simple efficient controllers increases efficiency from $33.1 \%$ to $77.4 \%$ for the Rise Time.

Theoretically, the Fall Time shall also be reduced by removing the residue inside the MR fluid brake quickly through a reverse current flow for $\Delta o f f$. The actual results of the test did not confirm the decrease in the fall time due to the use of the simple efficient control.

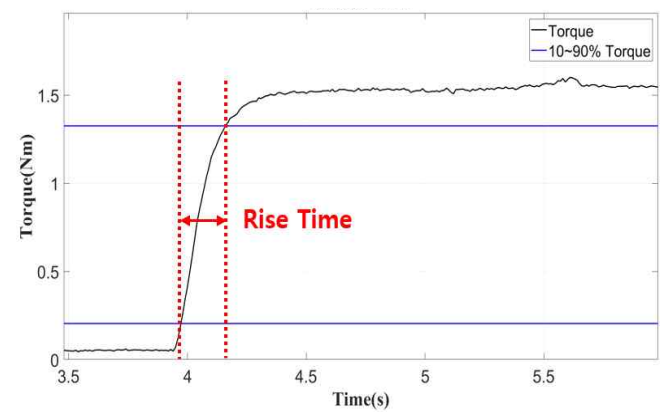

(a) General-Switching-Controller RiseTime_0.2A

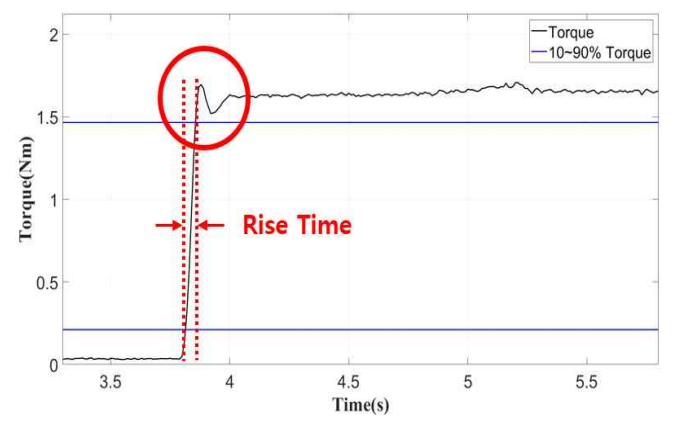

(c) Simple-Efficient-Controller RiseTime_0.2A

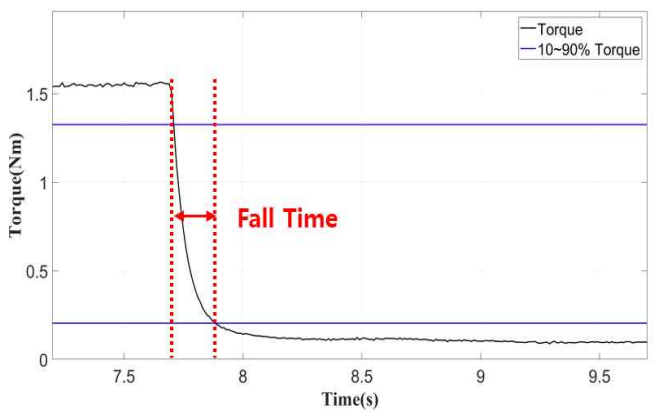

(b) General-Switching-Controller FallTime_0.2A

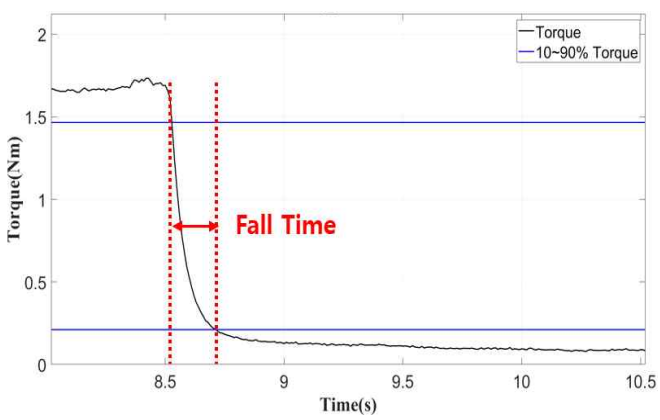

(d) Simple-Efficient-Controller FallTime_0.2A

[Fig. 5] Rise \& Fall Time when Entering Rated Voltage 
Development of a Simple Efficient Controller to Improve Respanse of MR Fluid Brakes

[Table 1] Response Characteristics of Simple Efficient Controller with Different Input Currents

\begin{tabular}{|c|c|c|c|c|c|c|}
\hline & & & \multicolumn{2}{|c|}{ General-Switching-Controller } & \multicolumn{2}{|c|}{ Simple-Efficient-Controller } \\
\hline $\begin{array}{c}\text { Current } \\
(\mathrm{A})\end{array}$ & $\begin{array}{c}\mathrm{VN} \\
(\mathrm{V})\end{array}$ & $\begin{array}{c}\mathrm{VH} \\
(\mathrm{V})\end{array}$ & $\begin{array}{c}\text { Rise Time } \\
(\mathrm{ms})\end{array}$ & $\begin{array}{c}\text { Fall Time } \\
(\mathrm{ms})\end{array}$ & $\begin{array}{c}\text { Rise Time } \\
(\mathrm{ms})\end{array}$ & $\begin{array}{c}\text { Fall Time } \\
(\mathrm{ms})\end{array}$ \\
\hline 0.2 & 4.6 & 32 & 186.9 & 173.4 & 42.2 & 175.2 \\
\hline 0.4 & 9.2 & 32 & 84.1 & 154 & 29.4 & 158.6 \\
\hline 0.6 & 13.8 & 32 & 52.7 & 154.9 & 24.9 & 153.2 \\
\hline 0.8 & 18.4 & 32 & 39.1 & 150.9 & 23.1 & 156.4 \\
\hline 1 & 23 & 32 & 34.4 & 145.8 & 23 & 149.5 \\
\hline
\end{tabular}

\section{Conclusion}

In this paper, our goal is to develop a simple efficient controller to improve the torque generation efficiency of MR fluid brakes, which varies according to the applied current. The comparisons and analyses were carried out in regard to the response characteristics of the simple efficient controller according to various input currents.

The General-Switching-Controller experiment was conducted simply by switching the rated voltage on-off. The Simple-Efficient-Controller experiment was conducted with different voltages applied over time, such as VH, VN, and VL. The efficiency of the Simple-Efficient-Controller was demonstrated by comparing the Rise Time and Fall Time with the two experiments.

Through this paper's proposed experiment, we observed a decrease in the Rise Time and an increase of torque generation efficiency between $33.1 \%$ and $77.4 \%$ by using the simple efficient controller. The reason why efficiency decreased as the input current value increased was due to the difference between $\mathrm{VH}$ and $\mathrm{VN}$, which had decreased due to the limitation of the $\mathrm{VH}^{\prime} \mathrm{s}$ availability in the power supply.

In addition, theoretically the Fall Time should be reduced using the controller by rapidly removing residues from the circuit through reverse current flow during $\triangle$ off. However, the decrease in Fall Time was not confirmed by the actual test. It will later confirm the Fall Time reduction through modification and supplementation of the circuit.

The experiments and analyses carried out in this paper have shown that the reaction of MR fluid brakes can be further improved by controlling the input voltage over time when the MR fluid brakes are applied. Based on these results, the applicability of MR fluid brakes could be again identified as actuators where immediate response is important. In the future, experiments will be carried out in various voltage environments. And it is thought that additional efficiency 
will be required in relation to the reduction of the Fall Time that the results of this experiment have not confirmed.

\section{Acknowledgement}

This development project was supported by the National Research Foundation of Korea (NRF-2019R1A2C2010195)

\section{References}

[1] Kim J. S., Ahn K. K., Ahn Y. K., Propose, Design and Control of a New Actuator Using MR Fluid, KSPE 2006 Spring Conference, (2006), May 18-19; Gyeongju, Korea.

[2] Im S. H., Min S. J, Hong J. P., Multi-Magnet Shape Design of a Highly Efficient Permanent Magnet Actuator for Wide Driving Temperature Range, KSME 2015 Autumn Conference, (2015), May 8; Busan, Korea.

[3] Kim H. R., Lee S. G., Park J. D., Kim. H., Choi M. J., Ryu J. K., Development of a Multi-Disk Type MR Fluid Brake with Modular Structure, Korean Society for Precision Engineering, (2018), Vol.35, No.2, pp.189-196.

[4] Hwang I. J., Weon I. S., Kim Y. J., Woo S. H., Lee S. G., Ryu J. K., Weon B. J., Experimentation and analysis on the effect of Chopper control on torque response characteristics of MR brake, The 34th ICROS Conference, (2019), May 16-18; Gyeongju, Korea.

[5] Kang B. H., Han C. H., Choi. S. B., Design and Control of MR Damper for Aircraft Landing Gear with Force Controller, KSNVE 2019 Spring Conference, (2019), February 20-23; Pyeongchang, Korea.

[6] Park Y. S., Lee M. K., Kim S. H., Design and Control of a Linear MR Brake, KSPE 2017 Autumn Conference, (2017), December 13-15; Gwangju, Korea.

[7] Jang H. S., Lee K. S., The Experiment on PID Controller and State Feedback Controller using Rotational Inverted Pendulum, The 42th KIEE Conference, (2011), July 20-22; Yongpyong, Korea.

[8] Lim C. Y., Seo K. Y., Comparison of Response Properties Determined in Two Torque Control Methods for a 2.75-MW Wind Turbine Under Turbulence Wind Speed, Transactions of the KSME, A, (2010), Vol.34, No.12, pp.1885-1891.

[9] Gang H. G., Choi S. B., Sohn J. W., Torque Tracking Control of MR Brake Using a Fuzzy-PID Control Algorithm, Transactions of the Korean Society for Noise and Vibration Engineering (Transactions of the KSNVE), (2017), Vol.27, No.4, pp.444-450.

[10] Youn D. W., Park J. H., Choi S. K., Yun S. N., Ham S. Y., Electromagnetic Analysis and design of rotary MR brake, KSPSE 2006 Autumn Conference, (2006), November 30-December 1; Busan, Korea.

[11] Park J. H., Ham Y. B., Yun S. N., Sea W. S., Basic Characteristic of MR Fluid and Its Application, 
Development of a Simple Efficient Controller to Improve Response of MR Fluid Brakes

Journal of Drive and Control (J. Drive Control)1, (2008), Vol.5, No.3, pp.9-14.

[12] Park J. H., Kim J. G., Yun D. W., Ham S. Y., Noh J. H., Yoo J. S., A Study on Dynamic Characteristics of High-Torque MR Brake, KSPE 2007 Spring Conference, (2007), June 20-22; Jeju, Korea. 